\title{
GOLD NANOPARTICLES-INDUCED ENHANCEMENT OF THE ANALYTICAL RESPONSE OF AN ELECTROCHEMICAL BIOSENSOR BASED ON A NEW ORGANIC-INORGANIC HYBRID COMPOSITE MATERIAL
}

\author{
$\underline{\text { M. Barbadillo Pérez de Ayala }}^{a^{*}}$, E. Casero $^{a}$, M.D. Petit-Domínguez ${ }^{a}$, F. Pariente ${ }^{a}$, E. \\ Lorenzo $^{a}$, L. Vázquez ${ }^{b}$ \\ ${ }^{a}$ Departamento de Química Analítica y Análisis Instrumental. Universidad Autónoma de \\ Madrid. Campus de Cantoblanco. 28049 Madrid. Spain. \\ ${ }^{b}$ Instituto de Ciencia de Materiales de Madrid (CSIC). 28049 Madrid. Spain. \\ *e-mail:martabpda@hotmail.com
}

Design and development of sensors based on enzyme immobilization onto transducer surfaces is of great interest in the field of bionanotechnology, mainly due to its potential applications in the development of analytical devices. In general, the retention of the enzymatic activity after the immobilization process is one of the most important issues in this field because the interaction between the enzyme and the solid surface can induce conformational changes in the adsorbed enzymes. The alteration of the three-dimensional conformation leads to a diminution of the catalytic activity. In this sense, the use of a polymeric network generated by sol-gel technology can be used as a strategy to provide a biocompatible environment for enzyme protection exhibiting additional advantages such as simplicity of preparation, physical rigidity, renewable surface and tuneable properties [1-2]. Moreover, the integration of nanomaterials into (bio)sensors has become an attractive method for improving the properties of the resulting analytical device. From a practical point of view, gold nanoparticles (AuNPs) are widely used due to their interesting physicochemical properties, including i) a large surface area, ii) an excellent biocompatibility and iii) highly attractive catalytic properties toward several compounds as a consequence of an enhancement of the electrical communication with the electrode surface [3]. These characteristics made this nanomaterial suitable to immobilize biomolecules, particularly enzymes, allowing the retention of the biological activity for a long period of time.

In the present work, we describe the preparation and characterization of a new organic-inorganic hybrid composite material from a three dimensional silica polymer network, obtained by means of the sol-gel technology using tetraethoxysilane (TEOS) as precursor. The final properties of the sol-gel matrix play a key role in the biosensor performance and can be easily controlled by varying some parameters such as the precursor type or the preparation conditions ( $\mathrm{pH}$, ratio of compounds, etc). This matrix provides an excellent network allowing the encapsulation of the different biosensor components: a biosensing molecule (glucose oxidase, GOx was chosen as a model), gold nanoparticles (AuNPs) and graphite powder (C). The resulting sol-gel / glucose oxidase / gold nanoparticles / graphite powder (TEOS/GOx/Au-NPs/C) hybrid material benefits from the advantages of their different components, giving rise to a material particularly attractive for electrochemical biosensor preparation. 
We have used as a surface characterization technique the Atomic Force Microscopy (AFM) since it allows us to assess the influence of the nanoparticle addition on the electrode nanomorphology [4-5]. Thus, we have studied by AFM operating in tapping mode in ambient conditions the surface morphology of the different systems despite the micro-roughness of some of them. We have measured areas from $64 \mathrm{~mm}^{2}$ down to $0.25 \mathrm{~mm}^{2}$ in order to characterize their roughness at the nanoscale. For the carbon-based electrodes, we addressed special emphasis to resolve the surface structure at atomic resolution. In contrast, for the enzyme-containing systems AFM was used to map the protein surface coverage and homogeneity. Figures A and B show AFM images of relative large and small areas, respectively, of the surface of a TEOS/GOx/Au-NPs/C electrode. The rounded structures seen in B correspond to the globular GOx enzymes and aggregates covering the electrode surface. Phase-contrast images were also acquired in order to gain further sensitivity to be able to distinguish soft and hard nanodomains within the sampled areas. Finally, we also performed preliminary force curve analysis on the different systems.

Finally, the designed material was applied to the determination of glucose in presence of hydroxymethylferrocene (HMF) as a redox mediator. The system exhibits a clear electrocatalytic activity towards glucose (see Figure C), allowing its determination at $200 \mathrm{mV}$ versus SCE. with a good operational performance.

Acknowledgements: This work has been supported by Comunidad de Madrid/Universidad Autónoma de Madrid (Project N ${ }^{\circ}$ CCG08-UAM/SEM-4074 and the Ministerio de Ciencia e Innovación (Project No. CTQ2008-05775 and FIS2006-12253-C06-03).

\section{References:}

[1] Lev, O., Tsionsky, L., Ravinovich, V., Analytical Chemistry 67(1) (1995) 22A-30A.

[2] Dave, B.C., Dunn, B.,Valentine, J.S., Zink, J.I., Analytical Chemistry 66(22) (1994) 1120A-1127A.

[3] Fendler, J.H., (Ed.), “Nanoparticles and Nanostructured Films” (1998) Weinheim, VCH.]

[4] Chiorcea-Paquim, A.M., Pauliukaite, R., Brett, C.M.A., Oliveira-Brett, A.M., Biosensors and Bioelectronics 24 (2008) 297-305.

[5] Pauliukaite, R., Chiorcea-Paquim, A.M., Oliveira-Brett, A.M., Brett, C.M.A., Electrochimica Acta 52 (2006) 1-8.

\section{Figures:}
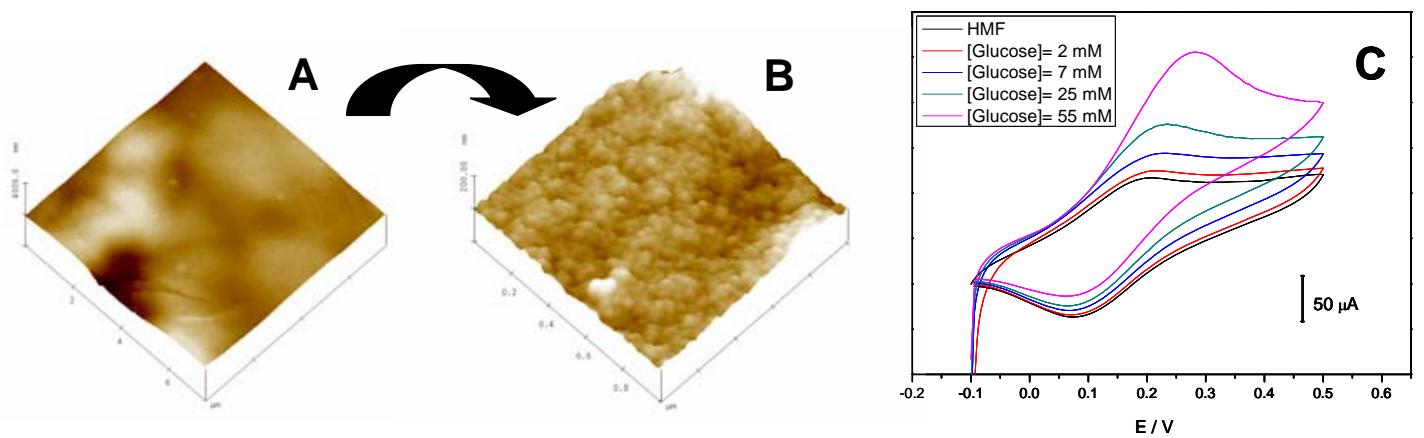